\title{
The Achilles Heel of Cervical Cancer: An Overlooked Target?
}

\section{Alfonso Dueñas-Gonzalez*}

Unit of Biomedical Research on Cancer, Institute of Biomedical Research, National Automous University of Mexico/National Cancer Institute, Mexico

Cervical cancer is the third most commonly diagnosed cancer worldwide and the fourth leading cause of cancer death in females, accounting for $9 \%(529,800)$ of total new cancer cases and $8 \%(275,100)$ of total cancer deaths among females in 2008. This cancer mainly affects socially disadvantaged women; hence, it is much more common in developing countries, where $83 \%$ of cases occur [1]. Despite these figures, there is a shortage of novel therapeutical agents for invasive cervical cancer in late stages of development.

In the current paradigm of molecular targeted therapy, cervical cancer has though not in the extension to common solid neoplasias such as breast, lung and colon- being subjected to exploration with whole genome and proteome tools to get a better understanding of its biology and hopefully to develop novel methods of diagnosis and treatment. A comprehensive genetic landscape analysis for cervical cancer has yet to be performed. Though preliminary, information from the Sanger data base [2] shows that the mutation frequencies in primary cervical cancer are: BRCA2 (8\%), FBXW7 (8\%), K-RAS (8\%), MSH2 (8\%), PI3KA (31\%), PTEN (8\%), RB1 (8\%), STK11 (23\%) and TP53 (23\%). These figures suggest that cervical cancer has not a high frequency of targetable gene alteration that could be translated into an effective treatment at to the present status of available drugs. More comprehensive studies are needed, however, the lessons we can take from whole genome sequencing of tumors are that most cancer types display substantial heterogeneity at the genetic level. For instances, individual solid organ tumors harbor on average $>50$ no silent clonal mutations in the coding regions of different genes (colorectal $=$ breast $>>$ pancreatic $>$ glioblastoma multiforme), and although a few of these genes are mutated in a high proportion of tumors, the prevalence at which the majority are mutated among different tumors of the same cancer type is low [3]. With the ever-increasing recognition that so-called "junk" DNA and intronic sequences contain functional elements, including regulatory regions and non coding RNAs, as revealed by The ENCODE Project Consortium [4] the total number of genomic alterations with functional consequences cannot be easily estimated. This underlies the complexity of the cancer genome landscape that confounds many of the original expectations of the cancer genome strategy. Thus, the future of personalized medicine concept where targeted therapies concurrent or sequential are to be administered based on patient stratification looks less hopeful than initially anticipated.

These by no means suggest that efforts toward an effective personalized medicine using targeted agents should not be continued but "common-sense driven or educated guess" approach need not to be overlooked. In this line, it is remarkable that Human Papilloma Virus (HPV) has long be established as the etiology of cervical cancer (and other neoplasias) by the regular presence of HPV DNA and their E6/ E7 viral oncogenes expression [5]. Of utmost importance are the solid evidence that cervical cancer cells require the expression of these viral oncogenes for maintaining the malignant phenotype via interaction of viral oncoproteins with growth-regulating host-cell proteins [610], thus, essentially, HPV oncoproteins directly or indirectly exert oncogenic effects upon cancer hallmarks [7], i) resisting cell death, ii) inducing angiogenesis, iii) sustaining proliferative signaling, iv) evading growth suppressors, v) activating invasion and metastasis, vi) and enabling replicative immortality.

The most compelling evidence on the key role of HPV oncoproteins in cervical cancer is the demonstration that repressing endogenous HPV oncogenes (E6/E7) mobilizes the p53 and Rb pathways in an orderly fashion to deliver growth inhibitory signals to the cells, hence unlike most cancers, in which the brakes on cell growth are broken, in HeLa cells -which are reminiscent of a advanced cervical cancer [11]- the driver is asleep, hence, down-regulation of E6/E7 is sufficient to wake up the driver and impose growth control [12]. This has subsequently being confirmed using a variety of nucleic acid-based [13-15] or small molecule approaches $[16,17]$. Nevertheless, despite the strong rationale of using anti-HPV targeted agents, clinical development of these agents against invasive cervical cancer is almost none.

Is there any scientific reason why to overlook HPV-oncoproteins as the long-searched therapeutic targets for invasive cervical cancer? Overlook means "to ignore deliberately or indulgently" Has it anything to do with the fact that current markets primarily drive the model of drug development? The question is open but it must be acknowledged that even though it is estimated that cervical cancer will decrease by $4 \%$ between 2010 and 2020 in seven major markets, 35,898 women will still develop cervical cancer in these countries.

\section{References}

1. Jemal A, Bray F, Center MM, Ferlay J, Ward E, et al. (2011) Global cancer statistics. CA Cancer J Clin 61: 69-90.

2. Dueñas-Gonzalez A, Cetina L, Coronel J, Cervantes-Madrid D (2012) Emerging drugs for cervical cancer. Expert Opin Emerg Drugs 17: 203-218.

3. Fox EJ, Salk JJ, Loeb LA (2009) Cancer genome sequencing--an interim analysis. 69: 4948-5490.

4. Bernstein BE, Birney E, Dunham I, Green ED, Gunter C, et al. (2012) An integrated encyclopedia of DNA elements in the human genome. Nature 489 $57-74$

5. zur Hausen J (2000) Papillomaviruses causing cancer: evasion from host-cel control in early events in carcinogenesis. J Natl Cancer Inst 92: 690-698.

6. Yugawa T, Kiyono T (2009) Molecular mechanisms of cervical carcinogenesis by high-risk human papillomaviruses: novel functions of E6 and E7 oncoproteins. Rev Med Virol 19: 97-113.

7. Wise-Draper TM, Wells SI (2008) Papillomavirus E6 and E7 proteins and their cellular targets. Front Biosci 13: 1003-1017.

*Corresponding author: Alfonso Dueñas-Gonzalez, Unit of Biomedical Research on Cancer, Institute of Biomedical Research, National Automous University of Mexico/National Cancer Institute, San Fernando 22, Tlalpan 14080, Mexico, Tel: +5255 54853034; E-mail: alfonso duenasg@yahoo.com

Received October 22, 2012; Accepted October 23, 2012; Published October 27, 2012

Citation: Dueñas-Gonzalez A (2012) The Achilles Heel of Cervical Cancer: An Overlooked Target? J Pharmacogenom Pharmacoproteomics 3: e128. doi:10.4172/2153-0645.1000e128

Copyright: ( 2012 Dueñas-Gonzalez A. This is an open-access article distributed under the terms of the Creative Commons Attribution License, which permits unrestricted use, distribution, and reproduction in any medium, provided the original author and source are credited. 
Citation: Dueñas-Gonzalez A (2012) The Achilles Heel of Cervical Cancer: An Overlooked Target? J Pharmacogenom Pharmacoproteomics 3:e128. doi:10.4172/2153-0645.1000e128

Page 2 of 8

8. Narisawa-Saito M, Kiyono T (2007) Basic mechanisms of high-risk human papillomavirus-induced carcinogenesis: roles of E6 and E7 proteins. Cancer Sci 98:1505-1511.

9. Moody CA, Laimins LA (2010) Human papillomavirus oncoproteins: pathways to transformation. Nat Rev Cancer 10: 550-560.

10. Hanahan D, Weinberg RA (2011) Hallmarks of cancer: the next generation. Cell 144: 646-674.

11. Goodwin EC, DiMaio D (2000) Repression of human papillomavirus oncogenes in HeLa cervical carcinoma cells causes the orderly reactivation of dormant tumor suppressor pathways. Proc Natl Acad Sci USA 97: 12513-1258.

12. Macville M, Schrock E, Padilla-Nash H, Keck C, Ghadimi BM, et al. (1999) Comprehensive and definitive molecular cytogenetic characterization of HeLa cells by spectral karyotyping. Cancer Res 59: 141-150.

13. Jiang M, Milner J (2002) Selective silencing of viral gene expression in HPVpositive human cervical carcinoma cells treated with siRNA, a primer of RNA interference. Oncogene 21: 6041-6048.
14. Sima N, Wang S, Wang W, Kong D, Xu Q, et al. (2007) Antisense targeting human papillomavirus type $16 \mathrm{E} 6$ and E7 genes contributes to apoptosis and senescence in SiHa cervical carcinoma cells. Gynecol Oncol 106: 299-304.

15. Gu W, Putral L, Hengst K, Minto K, Saunders NA, et al. (2006) Inhibition of cervical cancer cell growth in vitro and in vivo with lentiviral-vector delivered short hairpin RNA targeting human papillomavirus E6 and E7 oncogenes. Cancer Gene Ther 13: 1023-1032.

16. Mungala R, Kausar H, Munjal C, Gupta RC (2011) Withaferin A induces $p 53$ dependent apoptosis by repression of HPV oncogenes and upregulation of tumor suppressor proteins in human cervical cancer cells. Carcinogenesis 32 : 1697-1705.

17. Allen KL, Tschantz DR, Awad KS, Lynch WP, DeLucia AL (2007) A plant lignan, 3'-O-methyl-nordihydroguaiaretic acid, suppresses papillomavirus E6 protein function, stabilizes p53 protein, and induces apoptosis in cervical tumor cells. Mol Carcinog 46: 564-575. 